\title{
Intragastric Acidification Reduces the Occurrence of False-Negative Urea Breath Test Results in Patients Taking a Proton Pump Inhibitor
}

W. D. Chey, M.D., F.A.C.P., F.A.C.G., K. V. Chathadi, M.D., J. Montague, F. Ahmed, M.D., and

U. Murthy, M.D.

University of Michigan Health System, Ann Arbor, Michigan; and Syracuse Veterans Administration Medical Center, Syracuse, New York

OBJECTIVE: The aim of this study was to investigate whether reducing intragastric $\mathrm{pH}$, at the time of urea ingestion, decreases the likelihood of false-negative (FN) urea breath test (UBT) results in patients taking a proton pump inhibitor (PPI).

METHODS: Patients with active Helicobacter pylori infection underwent a baseline ${ }^{14} \mathrm{C}$-UBT (UBT-1) followed by treatment with lansoprazole $30 \mathrm{mg} /$ day for 14 to 16 days. On day 13, patients returned for a repeat standard UBT (UBT2). Between days 14 to 16 , patients underwent a modified UBT (UBT-3), which included consuming $200 \mathrm{ml}$ of $0.1 \mathrm{~N}$ citrate solution $30 \mathrm{~min}$ before and at the time of ${ }^{14} \mathrm{C}$-urea administration. Breath samples were collected 10 and 15 min after ${ }^{14} \mathrm{C}$-urea ingestion. Mean ${ }^{14} \mathrm{CO}_{2}$ excretion and the number of FN and equivocal UBT results were compared for the three UBTs.

RESULTS: A total of 20 patients completed the study. Lansoprazole caused a significant decrease in mean breath ${ }^{14} \mathrm{CO}_{2}$ excretion (disintegrations per minute) between UBT-1 (2.96 \pm 0.23$)$ and UBT-2 (2.08 $\pm 0.52, p<0.05)$. Lansoprazole caused six (30\%) FN and eight (40\%) equivocal UBT-2 results. Mean breath ${ }^{14} \mathrm{CO}_{2}$ excretion for UBT-3 $(677 \pm 514)$ was greater than for UBT-2 (234 \pm $327, p=0.001)$. UBT-3 caused only two (10\%) FN and three $(15 \%)$ equivocal results. The 15-min breath sample caused fewer FN and equivocal results than the 10-min sample for both UBT-2 and UBT-3.

CONCLUSIONS: Giving citrate before and at the time of ${ }^{14} \mathrm{C}$-urea administration increases mean breath ${ }^{14} \mathrm{CO}_{2}$ excretion and decreases FN and equivocal UBT results in patients taking a PPI. These observations suggest that it may be possible to design a UBT protocol that will remain accurate in the face of PPI therapy. (Am J Gastroenterol 2001;96: 1028-1032. ( ) 2001 by Am. Coll. of Gastroenterology)

Part of this work was presented at Digestive Diseases Week, San Diego, California, May, 2000.

\section{INTRODUCTION}

Recent guidelines support the use of the "test-and-treat" strategy for Helicobacter pylori (H. pylori) as initial management for young patients with uninvestigated dyspepsia $(1,2)$. In this strategy, patients with dyspepsia who are under the age of $50 \mathrm{yr}$ and have no "warning signs" are subjected to a nonendoscopic test for $H$. pylori. Those with evidence of infection are treated with a course of therapy directed at $H$. pylori. Recent studies suggest that this strategy reduces endoscopy workload without adversely affecting clinical outcomes $(3,4)$. With few exceptions, because of cost and convenience for the patient, nonendoscopic tests are also favored over endoscopic tests as a means of confirming $H$. pylori eradication.

The nonendoscopic tests for $H$. pylori can be split into those that identify an antibody response to this infection and those that identify active infection ("active tests"). Tests that identify active infection include the nonendoscopic urease tests $\left[{ }^{13} \mathrm{C}\right.$ and ${ }^{14} \mathrm{C}$-urea breath tests (UBT) and ${ }^{13} \mathrm{C}$-urea blood test] and the stool antigen test. Tests that identify active $H$. pylori infection have consistently been found to have sensitivity and specificity exceeding $90 \%(5,6)$.

A number of factors have been found to affect the accuracy of active testing. The recent use of antibiotics or bismuth-containing compounds can lead to false-negative (FN) test results (7). Use of a proton pump inhibitor (PPI) before the UBT has also been shown to cause FN results in up to $40 \%$ of patients $(8-10)$. For this reason, it is currently recommended that PPIs be withheld for 1 to $2 \mathrm{wk}$ before the UBT. Unfortunately, this recommendation is impractical for patients with acid-mediated disorders who often suffer with significant symptoms when their PPI is withheld.

In the current study, we attempted to confirm that PPIs decrease the sensitivity of the UBT. Previous work $(9,11)$ has suggested that PPIs induce FN UBT results by a $\mathrm{pH}-$ dependent mechanism. To test this hypothesis, we investigated whether the iatrogenic manipulation of intragastric $\mathrm{pH}$, at the time of urea ingestion, can reduce the likelihood of FN results in patients taking a PPI. 


\section{MATERIALS AND METHODS}

\section{Study Population}

Patients with active $H$. pylori infection as established by endoscopic testing (Raid urease testing [Pyloritek, Serim Research, Elkhart, IN] or histology) or urea breath testing were recruited from the outpatient Gastroenterology Clinics at two medical centers (University of Michigan Health System, Ann Arbor, MI, and Veterans Administration Medical Center, Syracuse, NY). All subjects were $>18$ yr old and gave written informed consent. All subjects were in good general health. Women of childbearing age were excluded from this protocol. None of the subjects had undergone previous gastric surgery. Those with a recent history of complicated peptic ulcer disease (upper GI bleeding, obstruction, or perforation) or grade III-IV endoscopic esophagitis were also excluded from the protocol. This protocol was approved by the Institutional Review Boards of the University of Michigan and Veterans Administration Medical Center.

\section{Study Protocol}

Eligible patients with active $H$. pylori infection underwent a baseline ${ }^{14} \mathrm{C}$-urea breath test (UBT-1, Pytest, Ballard Medical Products, Draper, UT) according to instructions provided by the manufacturer. After an overnight fast, patients received a capsule containing $1 \mu \mathrm{Ci}$ of ${ }^{14} \mathrm{C}$-urea. Breath samples were collected 10 and 15 min after the administration of ${ }^{14} \mathrm{C}$-urea and analyzed using a calibrated scintillation counter at each of the participating study sites. Appropriate quality control measures were carried out to assure the accuracy of the scintillation counter at each study site. A positive test was defined as a ${ }^{14} \mathrm{CO}_{2}$ excretion $\geq 200$ disintegrations per minute (DPM). A negative test was defined as a ${ }^{14} \mathrm{CO}_{2}$ excretion $\leq 50$ DPM. An equivocal study was defined as a ${ }^{14} \mathrm{CO}_{2}$ excretion $>50$ but $<200$ DPM. Patients with a negative or equivocal UBT-1 result were excluded from further analysis.

Patients with a positive UBT- 1 result were then treated with lansoprazole $30 \mathrm{mg}$ each morning 15 to $30 \mathrm{~min}$ before breakfast for 14 to 16 days. On day 13, patients underwent a second UBT (UBT-2) using the standard protocol provided by the manufacturer. Between days 14 to 16 , patients underwent a modified UBT (UBT-3). In the modified UBT, patients consumed $200 \mathrm{ml}$ of $0.1 \mathrm{~N}$ citrate solution $30 \mathrm{~min}$ before and at the time of ${ }^{14} \mathrm{C}$-urea ingestion. This dosage of citrate has been suggested to be the optimal test meal for the ${ }^{13} \mathrm{C}$-UBT (12).

\section{Statistical Analysis}

Mean ${ }^{14} \mathrm{CO}_{2}$ excretions (DPM) with UBT-2 and UBT-3 (mean $\pm \mathrm{SDM}$ ) were compared to UBT-1 using a one-way analysis of variance for repeated measures using transformed data (natural log scale) for heterogeneous variability within a group and between groups. The Bonferroni $t$ test was used as a posthoc test. A $p$ value of $<0.05$ defined a statistically significant difference between values. In addi-
Table 1. The 15-min ${ }^{14} \mathrm{CO}_{2}$ Excretion (DPM) for UBT-1, UBT2 , and UBT-3

\begin{tabular}{|c|c|c|c|c|c|c|}
\hline \multirow[b]{2}{*}{ Subject } & \multicolumn{2}{|c|}{ UBT-1 } & \multicolumn{2}{|c|}{ UBT-2 } & \multicolumn{2}{|c|}{ UBT-3 } \\
\hline & Abs & $\log$ & Abs & $\log$ & Abs & $\log$ \\
\hline 1 & 416 & 2.62 & 11 & 1.04 & 10 & 1 \\
\hline 2 & 496 & 2.7 & 44 & 1.64 & 43 & 1.63 \\
\hline 3 & 371 & 2.57 & 73 & 1.86 & 96 & 1.98 \\
\hline 4 & 1264 & 3.1 & 20 & 1.3 & 180 & 2.26 \\
\hline 5 & 1793 & 3.25 & 406 & 2.61 & 194 & 2.29 \\
\hline 6 & 1299 & 3.11 & 150 & 2.18 & 267 & 2.43 \\
\hline 7 & 571 & 2.76 & 50 & 1.7 & 428 & 2.63 \\
\hline 8 & 500 & 2.7 & 84 & 1.92 & 446 & 2.65 \\
\hline 9 & 860 & 2.93 & 89 & 1.95 & 446 & 2.65 \\
\hline 10 & 877 & 2.94 & 115 & 2.06 & 536 & 2.73 \\
\hline 11 & 1034 & 3.02 & 154 & 2.19 & 560 & 2.75 \\
\hline 12 & 795 & 2.9 & 131 & 2.12 & 636 & 2.8 \\
\hline 13 & 992 & 3 & 50 & 1.7 & 889 & 2.95 \\
\hline 14 & 1560 & 3.19 & 1382 & 3.14 & 905 & 2.96 \\
\hline 15 & 898 & 2.95 & 45 & 1.65 & 1012 & 3.01 \\
\hline 16 & 1167 & 3.07 & 663 & 2.82 & 1016 & 3.01 \\
\hline 17 & 958 & 2.98 & 208 & 2.32 & 1254 & 3.1 \\
\hline 18 & 2226 & 3.35 & 167 & 2.22 & 1375 & 3.14 \\
\hline 19 & 542 & 2.73 & 628 & 2.8 & 1469 & 3.17 \\
\hline 20 & 2086 & 3.32 & 216 & 2.33 & 1784 & 3.25 \\
\hline Mean & 1035 & 2.96 & 234 & 2.08 & 677 & 2.62 \\
\hline SDM & 543 & 0.23 & 264 & 0.52 & 515 & 0.56 \\
\hline
\end{tabular}

Absolute data (Abs) and data after log transformation (Log) followed by statistical comparison using the Bonferroni $t$-test.

$\mathrm{DPM}=$ disintegrations per minute; $\mathrm{UBT}=$ urea breath test.

tion, the absolute number of $\mathrm{FN}$ or equivocal results yielded by UBT-2 and UBT-3 were compared using UBT- 1 as a gold standard.

\section{RESULTS}

A total of 21 (19 men and two women, mean age $=55 \pm$ $12 \mathrm{yr}$ ) patients enrolled in this protocol. One patient was excluded from the final analysis because of an equivocal UBT-1 result. The remaining 20 patients had a positive UBT-1 result as defined as a ${ }^{14} \mathrm{CO}_{2}$ excretion $\geq 200$ DPM. All patients reported $>90 \%$ compliance with lansoprazole therapy at their follow-up visits. Results from UBT-1, UBT-2, and UBT-3 can be found in Table 1 and Figure 1.

\section{Effect of Lansoprazole on Mean Breath ${ }^{14} \mathrm{CO}_{2}$ Excretion and Sensitivity of UBT-2}

Lansoprazole led to a highly significant decrease in the mean breath ${ }^{14} \mathrm{CO}_{2}$ excretion between UBT-1 (2.96 \pm 0.23 DPM) and UBT-2 (2.08 \pm 0.52 DPM, $p<0.05)$. Using results obtained from the 15 -min breath collection, lansoprazole caused six (30\%) FN and eight (40\%) equivocal UBT-2 results.

\section{Effect of Lansoprazole on Mean Breath ${ }^{14} \mathrm{CO}_{2}$ Excretion and Sensitivity of UBT-3}

Mean breath ${ }^{14} \mathrm{CO}_{2}$ excretion for the modified UBT-3 was not significantly lower than that yielded by UBT-1 $(p>$ 0.05). On the other hand, mean breath ${ }^{14} \mathrm{CO}_{2}$ excretion for 


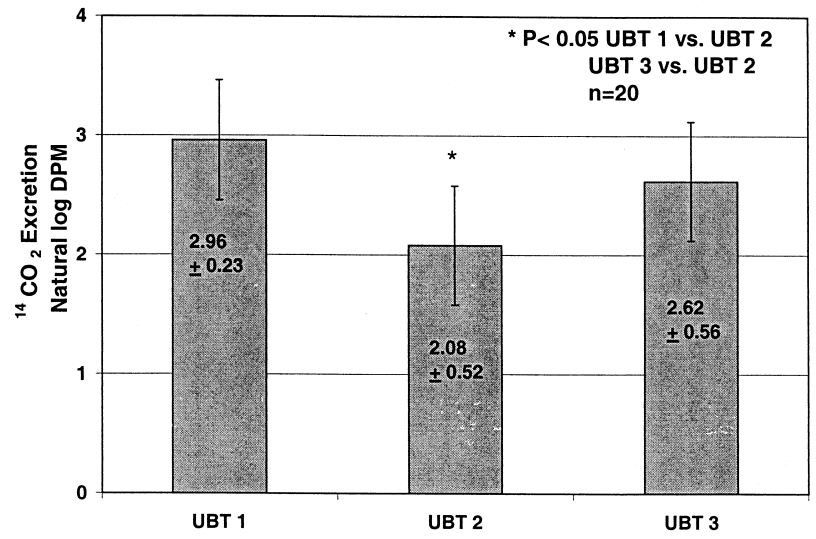

Figure 1. Effect of lansoprazole on mean breath ${ }^{14} \mathrm{CO}_{2}$ excretion for UBT-1, UBT-2, and UBT-3 using log-transformed data.

the modified UBT-3 (2.62 \pm 0.56 DPM) was significantly greater than for UBT-2 (2.08 \pm 0.52 DPM, $p<0.05)$. When results from the 15-min breath collection were used, the modified UBT-3 caused only two (10\%) FN and three (15\%) equivocal results (Fig. 2).

\section{Optimal Timing of Breath Sample Collection}

Results obtained from the 10- and 15-min breath collections were compared. There was no significant difference between mean 10- and 15-min breath ${ }^{14} \mathrm{CO}_{2}$ excretions obtained during each of the three breath tests. However, the 15-min breath sample caused fewer FN and equivocal results than the 10-min breath collection for both UBT-2 and UBT-3 (Fig. 2).

\section{DISCUSSION}

In this age of cost-conscious medicine, nonendoscopic tests for $H$. pylori have taken on great importance both as a means of primary diagnostic testing and to establish cure after antimicrobial treatment. Studies have found that the recent use of PPIs can affect the sensitivity of the ${ }^{14} \mathrm{C}$-UBT or

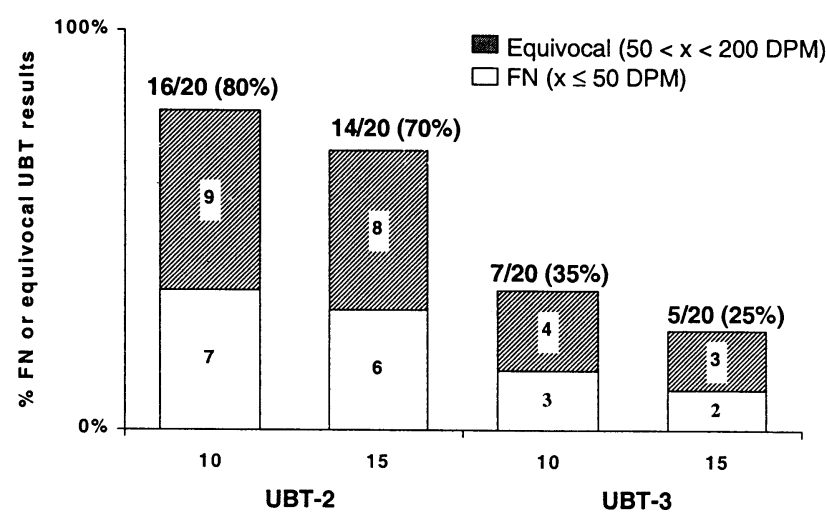

Figure 2. Effect of citrate on the rate of $\mathrm{FN}$ and equivocal ${ }^{14} \mathrm{C}-\mathrm{UBT}$ results using the 10- and 15-min breath samples.
${ }^{13} \mathrm{C}$-UBT (8-10) and stool antigen test (13). Standard doses of omeprazole have been found to significantly reduce breath ${ }^{14} \mathrm{CO}_{2}$ excretion and to induce FN or equivocal UBT results in $38.5 \%$ of $H$. pylori-infected patients (8). This effect resolved within 5 days of discontinuing omeprazole. Laine et al. (10) reported that standard doses of lansoprazole induced $\mathrm{FN}{ }^{13} \mathrm{C}$-UBT results in $33 \%$ of patients and that this effect lasted for 7 to 14 days. On the basis of these observations, most experts now recommend that PPIs be withheld for 7 to 14 days before the UBT.

Several mechanisms have been proposed to explain the ability of PPIs to induce FN UBT results (9). Both omeprazole and lansoprazole have a mean inhibitory concentration for $H$. pylori (14). McGowan et al. reported that PPIs inhibit H. pylori growth at low $\mathrm{pH}$ through a urease-independent mechanism suggesting a true "antibiotic" effect, perhaps as a consequence of effects on bacterial proton pumps (15). Another possibility is that the significant increase in intragastric $\mathrm{pH}$ developed by many patients treated with PPIs allows overgrowth of the stomach with other species of bacteria (16). It is possible that this bacterial overgrowth could inhibit the growth or metabolic activity of $H$. pylori.

Urease is an enzyme produced in large quantities by $H$. pylori. The metabolic activity of this enzyme provides the basis for the UBT. Recent reports have led to an improved understanding of the functional importance of urease (1719). H. pylori can survive from $\mathrm{pH} 4$ to 8.5. In the absence of urea, H. pylori cannot survive below an external $\mathrm{pH}$ of 4 . In the presence of urease and its substrate, urea, $H$. pylori can survive in an external $\mathrm{pH}$ as low as 2.5 (18). Urease has also been found to be essential to $H$. pylori colonization in mammals (20). Two isoforms of the enzyme have been described, external and internal urease. External urease is believed to arise largely as a consequence of cell lysis (18). Internal urease is responsible for most of $H$. pylori's metabolic activity from $\mathrm{pH} 2.5$ to 6.5 . Internal urease plays a critical role in stabilizing periplasmic $\mathrm{pH}$ when external $\mathrm{pH}$ is low and accounts for $H$. pylori's relative acid resistance. However, when the environmental $\mathrm{pH}$ rises above 4 to 5.5, in vitro studies have demonstrated a corresponding rise in periplasmic $\mathrm{pH}$, resulting in a progressive decrease in internal urease activity (19). As such, it is conceivable that any medication with the ability to significantly raise intragastric $\mathrm{pH}$ could lead to FN results for clinical tests relying upon the identification of urease activity.

Several in vivo studies seem to corroborate the above in vitro observations. Using prolonged ambulatory intragastric $\mathrm{pH}$ monitoring, our group has previously demonstrated that patients with the greatest increase in intragastric $\mathrm{pH}$ while taking lansoprazole are the ones most likely to develop FN or equivocal ${ }^{14} \mathrm{C}$-UBT results. We made similar observations in patients taking high-dose ranitidine suggesting that elevated intragastric $\mathrm{pH}$, rather than something unique to PPIs, was responsible for inducing FN UBT results (9). In another recent study, Lew and et al. performed serial ${ }^{13} \mathrm{C}$ - 
UBTs after intragastric titration to $\mathrm{pH} 3,5$, and 7 (11). Two of 11 patients developed a FN UBT result when the intragastric $\mathrm{pH}$ was elevated to 7 .

In the current study, using a standard ${ }^{14} \mathrm{C}$-UBT protocol, we have found that lansoprazole $(30 \mathrm{mg} /$ day) significantly reduced mean breath ${ }^{14} \mathrm{CO}_{2}$ excretion and induced $\mathrm{FN}$ $(30 \%, \mathrm{n}=6)$ or equivocal $(40 \%, \mathrm{n}=8)$ results in the majority of patients. The administration of citrate before and at the time of ${ }^{14} \mathrm{C}$-urea ingestion significantly increased mean breath ${ }^{14} \mathrm{CO}_{2}$ excretion and decreased the number of FN or equivocal ${ }^{14} \mathrm{C}$-UBT results. These observations lend further support to the notion that PPIs induce FN UBT results by increasing intragastric $\mathrm{pH}$ and, consequently, decreasing $H$. pylori's urease activity. We have further found that a 15-min breath sample led to fewer FN and equivocal results than the traditional 10-min breath sample for either UBT-2 or UBT-3.

It is interesting that the addition of citrate reduced but did not eliminate FN and equivocal UBT results in patients taking a PPI. It is possible that the degree and/or duration of intragastric acidification employed in this study was not sufficient to reactivate urease in all of our study subjects. It is also possible that the effect of intragastric $\mathrm{pH}$ on $H$. pylori's urease activity does not provide a complete explanation for the ability of PPIs to induce FN UBT results. PPIs have been shown to decrease the density of $H$. pylori organisms in the antrum (21). Recent studies have also demonstrated an effect of PPIs on the accuracy of the stool antigen test, which does not rely upon the identification of urease (13). These observations suggest that PPIs affect $H$. pylori viability and bacterial load in addition to urease activity.

In conclusion, we have confirmed the deleterious effect of PPIs on the sensitivity of the ${ }^{14} \mathrm{C}$-UBT. We have also found that the use of citrate before and at the time of ${ }^{14} \mathrm{C}$-urea administration increases mean breath ${ }^{14} \mathrm{CO}_{2}$ excretion and decreases the likelihood of FN and equivocal results. These results suggest that the effects of PPIs on the UBT are, in large part, mediated through a $\mathrm{pH}$-dependent mechanism. These observations further suggest that it may be possible to design a UBT protocol that will remain accurate in the face of PPI therapy.

\section{ACKNOWLEDGMENTS}

This study was supported by unrestricted grants to the University of Michigan from TAP Pharmaceuticals and Ballard Medical Products.

Reprint requests and correspondence: William D. Chey, M.D., F.A.C.P., F.A.C.G., 3912 Taubman Center, Ann Arbor, MI 481090362.

Received Aug. 3, 2000; accepted Nov. 6, 2000.

\section{REFERENCES}

1. Talley NJ, Axon A, Bytzer P, et al. Management of uninvestigated and functional dyspepsia: A working party report for the World Congress of Gastroenterology 1998. Aliment Pharm Ther 1999;13:1135-48.

2. Talley NJ, Silverstien MD, Agreus L, et al. AGA technical review: Evaluation of dyspepsia. Gastroenterology 1998;114: $579-81$.

3. Lassen AT, Pederson FM, Bytzer P, et al. H. pylori test and treat or prompt endoscopy for dyspeptic patients in primary care. A randomized, controlled trial of two management strategies: One year follow-up. Gastroenterology 1998;114: A196 (abstract).

4. Heaney A, Collins JSA, Watson RGP, et al. A prospective randomized trial of a "test and treat" policy versus endoscopy based management in young Helicobacter pylori positive patients with ulcer-like dyspepsia, referred to a hospital clinic. Gut 1999;45:186-90.

5. Cutler AF, Havstad S, Ma CK, et al. Accuracy of invasive and noninvasive tests to diagnose Helicobacter pylori infection. Gastroenterology 1995;109:136-41.

6. Vaira D, Malfertheiner P, Megraud F, et al. Diagnosis of Helicobacter pylori infection with a new non-invasive antigen-based assay. HpSA European Study Group. Lancet 1999; 354:30-3.

7. Brown KE, Peura DA. Diagnosis of Helicobacter pylori infection. Gastroenterol Clin N Am 1993;22:105-15.

8. Chey WD, Spybrook M, Carpenter S, et al. Prolonged effect of omeprazole on the ${ }^{14} \mathrm{C}$-urea breath test. Am J Gastroenterol 1996;91:89-92.

9. Chey WD, Woods M, Scheiman JM, et al. Lansoprazole and ranitidine affect the accuracy of the 14C-urea breath test by a $\mathrm{pH}$ dependent mechanism. Am J Gastroenterol 1997;92:44650 .

10. Laine L, Estrada R, Trujillo M, et al. Effect of proton pump inhibitor therapy on diagnostic testing for Helicobacter pylori. Ann Intern Med 1998;129:547-50.

11. Lew EA, Pisegna JR, Ohning GV, et al. Intragastric pH titration affects the accuracy of 13C-urea breath test in the diagnosis of Helicobacter pylori. Gastroenterology 1999;116: A235 (abstract).

12. Leodolter A, Dominguez-Munoz E, von Arnim U, et al. Validity of a modified ${ }^{13} \mathrm{C}$-urea breath test for pre- and posttreatment diagnosis of Helicobacter pylori infection in the routine clinical setting. Am J Gastroenterol 1999;94:2100-4.

13. Bravo LE, Realpe JL, Campo C, et al. Effects of acid suppression and bismuth medications on the performance of diagnostic tests for Helicobacter pylori infection. Am J Gastroenterol 1999;94:2380-3.

14. Iwahi T, Satoh H, Nakao M, et al. Lansoprazole, a novel benzimidazole proton pump inhibitor, and its related compounds have selective activity against Helicobacter pylori. Antimicrob Agents Chemother 1991;35:490-6.

15. McGowan CC, Cover TL, Blaser MJ. The proton pump inhibitor omeprazole inhibits acid survival of Helicobacter pylori by a urease-independent mechanism. Gastroenterology 1994; 107:738-43.

16. Sharma BK, Santana IA, Wood EC, et al. Intragastric bacterial activity and nitrosation before, during, and after treatment with omeprazole. Br Med J 1984;289:717-9.

17. Meyer-Rosenberg K, Scott DR, Rex D, et al. The effect of environmental $\mathrm{pH}$ on the proton motive force of Helicobacter pylori. Gastroenterology 1996;111:886-900.

18. Scott DR, Weeks D, Hong C, et al. The role of internal urease in acid resistance of Helicobacter pylori. Gastroenterology 1998; 114:58-70. 
19. Rektorschek M, Weeks D, Sachs G, et al. Influence of pH on metabolism and urease activity of Helicobacter pylori. Gastroenterology 1998;115:628-41.

20. Tsuda M, Karita M, Morshed MG, et al. A urease-negative mutant of Helicobacter pylori constructed by allelic exchange mutagenesis lacks the ability to colonize the nude mouse stomach. Infect Immun 1994;62:3586-9.

21. Logan RPH, Walker MM, Misiewicz JJ, et al. Changes in the intragastric distribution of Helicobacter pylori during treatment with omeprazole. Gut 1995;36:12-6. 